\title{
Considerations of Mast Stability and Prevention of Disproportionate Collapse in the Event of Membrane Failure
}

\section{STRUCTURAL MEMBRANES 2021}

\author{
David M. Campbell P.E.
}

*Geiger Lynch MacBain Campbell Engineers, P.C.

2 Executive Blvd. Suite 309

Suffern, NY 10901 USA

Email: dmc@geigerengineers.com, web page: http://www.geigerengineers.com

\begin{abstract}
Commonly used tension membrane textile based materials have relatively low tear strength in relation to their tensile strength. This results in the membrane being vulnerable to tear propagation due imperfections, cuts, or damage when subjected to high service demands. In recognition of this, it has been good design practice is to consider the system consequences of the failure of tension membrane in membrane structures. This consideration has been codified in ASCE/SEI 55 Standard, a reference standard adopted by many IBC based codes in the USA. Specifically paragraph 4.3.2 states "Tension membrane structures shall be designed so that failure of the membrane, or of a single supporting element, does not result in a disproportionate collapse or compromise life safety."
\end{abstract}

The masts in mast supported membrane structures are elements that require special consideration in the event of a membrane failure as they are often components of considerable size and mass that rely upon the system including the tension membrane for stability.

This paper reviews the issues of stability of masts in post failure modes and various design solutions which have been adopted. Examples of built mast supported structures are used to illustrate design strategies, philosophies, and solutions.

\section{REFERENCES}

[1] ASCE/SEI 55-16 “Tensile Membrane Structures” American Society of Civil Engineers, ISBN 9780784414378 